\title{
Transferência do grande dorsal para lesões anterossuperiores irreparáveis do manguito rotador: descrição de técnica cirúrgica*
}

\section{Latissimus Dorsi Transfer to Irreparable Anterosuperior Lesions to the Rotator Cuff: Description of Surgical Technique}

\author{
Ivan Fadanelli Simionato ${ }^{1}$ Fábio Yoshihiro Matsumoto ${ }^{1(0)}$ Almiro Gerszon Britto ${ }^{10}$ \\ Marco Tonding Ferreira ${ }^{1}$ Augusto Heinen Eichenberg ${ }^{2}$ Fernando Carlos Mothes ${ }^{1}$ ()
}

${ }^{1}$ Grupo de Cirurgia do Ombro, Departamento de Ortopedia e

Endereço para correspondência Fernando Carlos Mothes, MD,

Traumatologia, Santa Casa de Porto Alegre, Porto Alegre, RJ, Brasil Departamento de Ortopedia e Traumatologia, Rua Professor Annes

2 Departamento de Ortopedia e Traumatologia, Clínica Conex, Ijuí, RS, Brasil

Dias, 295, Centro Histórico, Porto Alegre, RS, 90020-090, Brasil

(e-mail: fcmothes@yahoo.com.br).

Rev Bras Ortop 2021;56(5):671-674.

\section{Resumo \\ Palavras-chave \\ - ombro/cirurgia \\ - manguito rotador \\ - transferência tendinosa \\ - traumatismos dos tendões \\ As lesões do manguito rotador do ombro podem ter diversas configurações, e são divididas e/ou classificadas conforme o tamanho da lesão, o tendão envolvido, suas combinações, e se existe a possibilidade de reparo. As lesões irreparáveis do subesca- pular, assim como as lesões irreparáveis anterossuperiores, podem gerar disfunção do ombro devido à perda dos depressores da cabeça umeral, o que causa dor e limitação funcional. Entre as alternativas terapêuticas estão as transferências miotendíneas, sendo a técnica de transferência do peitoral maior para a tuberosidade menor a mais estudada e difundida nas últimas décadas. No entanto, alguns estudos anatômicos sugerem que o grande dorsal seja uma alternativa mais adequada para o tratamento dessas lesões por apresentar vetores de força mais parecidos com os do subescapular, além de apresentar resultados promissores nas avaliações clínicas de curto segui- mento. Este trabalho tem o objetivo de descrever a técnica cirúrgica desenvolvida para transferência do grande dorsal nas lesões irreparáveis do subescapular e anterossu- periores do manguito rotador.}

Trabalho desenvolvido no Departamento de Ortopedia e Traumatologia, Grupo de Cirurgia do Ombro da Santa Casa de Porto Alegre, Porto Alegre, RS, Brasil.

recebido

30 de Julho de 2020

aceito

28 de Outubro de 2020
DOI https://doi.org/

$10.1055 / \mathrm{s}-0041-1726060$ ISSN 0102-3616. (c) 2021. Sociedade Brasileira de Ortopedia e Traumatologia. All rights reserved.

This is an open access article published by Thieme under the terms of the Creative Commons Attribution-NonDerivative-NonCommercial-License, permitting copying and reproduction so long as the original work is given appropriate credit. Contents may not be used for commercial purposes, or adapted, remixed, transformed or built upon. (https://creativecommons.org/ licenses/by-nc-nd/4.0/)

Thieme Revinter Publicações Ltda., Rua do Matoso 170, Rio de Janeiro, RJ, CEP 20270-135, Brazil 


\author{
Abstract \\ Keywords \\ - shoulder/surgery \\ - rotator cuff \\ - tendon transfer \\ - tendon injuries
}

Lesions to the shoulder rotator cuff may have several configurations, and are divided and classified according to the size of the lesion, the tendon involved, its combinations, and whether there is possibility of repair. Irreparable subscapular lesions, as well as irreparable anterosuperior lesions, may generate shoulder dysfunction due to loss of humeral head depressants, causing pain and functional limitation. Myotendinous transfers are among the therapeutic alternatives, and the most studied and widespread in recent decades is the transfer of the pectoralis major to the minor tuberosity. However, some anatomical studies suggest that the latissimus dorsi is a more appropriate alternative for the treatment of these lesions, because it presents force vectors more similar to those of the subscapularis, besides presenting promising results in the clinical evaluations with short follow-up. The present work aims to describe the surgical technique developed for transfer of the latissimus dorsi in cases of irreparable subscapular and anterossuperior lesions to the rotator cuff.

\section{Introdução}

As lesões do manguito rotador do ombro têm diversas configurações, e são classificadas conforme o tamanho da lesão, os tendões envolvidos, e sua reparabilidade. ${ }^{1,2}$ As lesões iniciais do subescapular podem passar despercebidas clinicamente, o que ocasiona o atraso do tratamento e a evolução para uma lesão retraída associada à degeneração gordurosa do ventre muscular, que, assim, se torna irreparável ${ }^{1}$ Não é incomum sua associação com lesões da porção anterior do supraespinhal, que caracterizam o que se chama de lesões anterossuperiores. ${ }^{3}$ Nesses casos, observa-se perda dos depressores da cabeça umeral, o que leva a uma instabilidade anterossuperior que gera dor e disfunção no ombro. ${ }^{3}$

As alternativas de tratamento cirúrgico para lesões irreparáveis do subescapular incluem as transferências musculares, como as do peitoral maior, do peitoral menor, e do grande dorsal ${ }^{2,4}$ Já para os pacientes que apresentam o quadro associado à osteoartrite glenoumeral, a artroplastia reversa deve ser a alternativa, pois atua tanto na resolução da degeneração articular quanto no tratamento da disfunção decorrente da lesão do manguito. ${ }^{4}$

A técnica mais estudada e difundida para o tratamento das lesões irreparáveis do subescapular é a transferência do peitoral maior para a tuberosidade menor, que apresenta resultados clínicos favoráveis e mantidos em longo prazo $^{1,5-7}$ No entanto, estudos anatômicos mostram que o tendão do grande dorsal apresenta um vetor mais próximo ao do subescapular, por também se originar da parede posterior do tórax, diferentemente do peitoral maior, que se origina da parede anterior e apresenta vetor de força quase ortogonal ao da porção anterior do manguito rotador. $^{8} \mathrm{Em}$ um estudo anatômico em cadáveres, Elhassan et al. ${ }^{8}$ demonstraram que a transferência do grande dorsal em casos de lesões do subescapular é anatomicamente possível e com baixo risco de lesão nervosa. Dessa forma, a proposta deste trabalho é descrever a técnica cirúrgica desenvolvida pelo Grupo de Cirurgia do Ombro para transferência do grande dorsal nas lesões irreparáveis do subescapular e/ou lesões anterossuperiores irreparáveis do manguito rotador.

\section{Descrição da Técnica}

Após realizar bloqueio regional interescalênico e anestesia geral, o paciente é posicionado em cadeira de praia com auxílio de apoio para o cotovelo. A abordagem deltopeitoral é realizada com incisão de aproximadamente $7 \mathrm{~cm}$ na região anterior do ombro para a identificação da lesão irreparável do subescapular. O ligamento coracoacromial é preservado para evitar a migração anterossuperior da cabeça umeral e, se o cabo longo do bíceps estiver íntegro, a tenotomia com ou sem tenodese é realizada. O tendão do grande dorsal é identificado em posição distal ao subescapular, medial ao cabo longo do bíceps, e posterior ao peitoral maior. A tenotomia de $2 \mathrm{~cm}$ proximais do peitoral maior é efetuada (-Figura 1) para exposição adequada da porção distal do grande dorsal, que muitas vezes tem uma inserção arciforme (-Figura 2). Em seguida, descola-se o tendão do grande dorsal do redondo maior com auxílio de um descolador de freer. Essa etapa é importante, pois eventualmente os tendões têm uma inserção ou fáscia quase conjunta, e se o grande dorsal não for bem liberado do redondo maior,

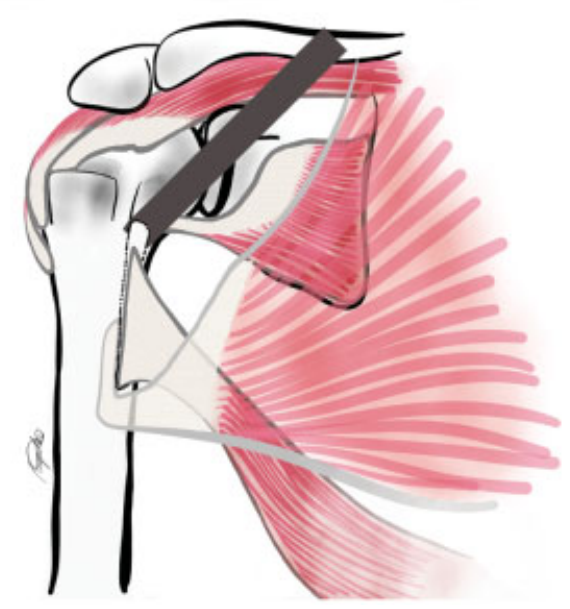

Fig. 1 Desenho da lesão anterossuperior irreparável com tenotomia do peitoral maior para exposição adequada do grande dorsal (GD) e a sua coleta com um chip ósseo. 


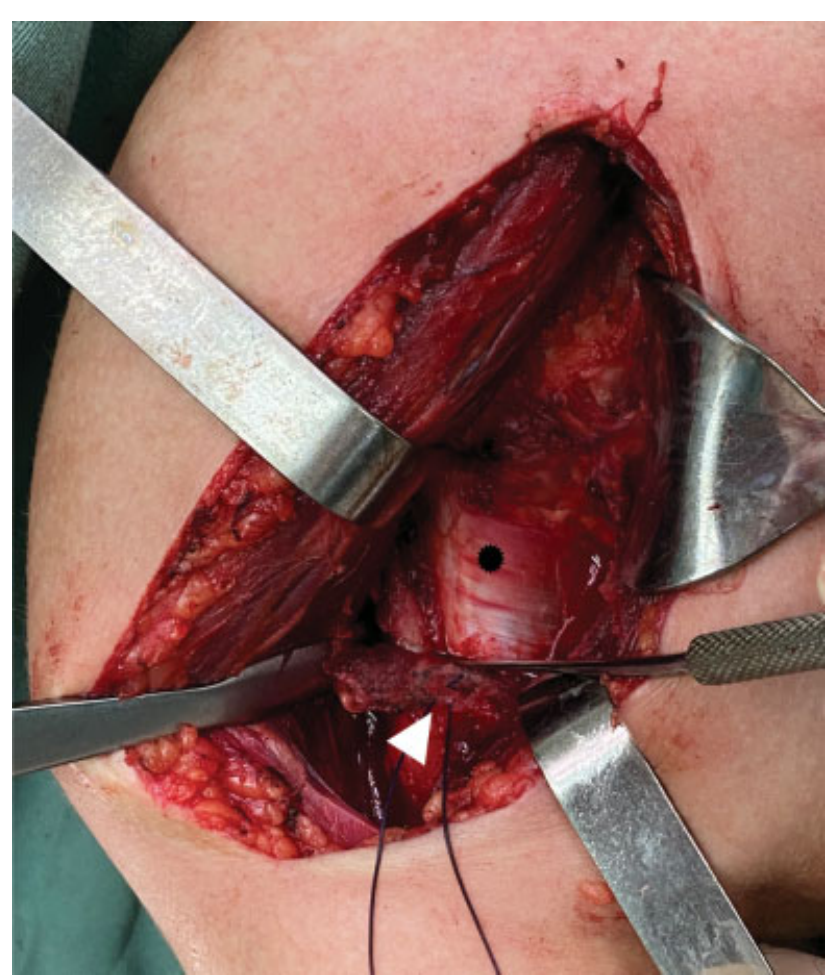

Fig. 2 Foto transoperatória de via deltopeitoral da porção distal do GD (asterisco preto) com o peitoral maior tenotomizado (seta branca).

dificultará sua excursão proximal até a cabeça do úmero. Em seguida, é feita a desinserção do grande dorsal do úmero, por meio de um osteótomo delicado e afiado, e o preparo de um chip ósseo na sua porção proximal, com cuidado para não violar a inserção do redondo maior (- Figura 1). Segue-se a coleta do tendão usando um bisturi de lâmina 15 adjacente ao osso, para não haver perda do enxerto. Nesse passo de coleta do enxerto, é importante que a porção distal da inserção do grande dorsal seja visualizada adequadamente para que não se finalize a coleta antes do seu final e, por consequência, se realize uma amputação da parte distal do enxerto, o que pode dificultar a excursão e qualidade do tendão no momento da inserção ( - Figura 2). depois, realizase o reparo do tendão do grande dorsal com dois pontos do tipo Krakow com fios inabsorvíveis (Ethibond 5, Ethicon, Inc., Cincinnati, Ohio, EUA) em cada borda do tendão, deixando a parte central livre, evitando algum prejuízo na vascularização e no potencial de cicatrização (-Figura 3). Em seguida, realiza-se a liberação da parte muscular do enxerto com uma rugina romba do tipo Cobb para melhorar a excursão e o comprimento do tendão, cuidando-se para não colocar em risco as estruturas neurovasculares que se encontram mediais e anteriores ao grande dorsal, como o nervo radial. No próximo passo, testa-se a excursão do grande dorsal e delimita-se o ponto de inserção mais proximal. Então, o tubérculo menor e a porção mais anterior do tubérculo maior (nas lesões anterossuperiores) são decorticados com um saca-bocado, e é realizada a transferência do enxerto do grande dorsal e sua fixação com dois pontos transósseos no ponto mais proximal do leito decorticado do tubérculo

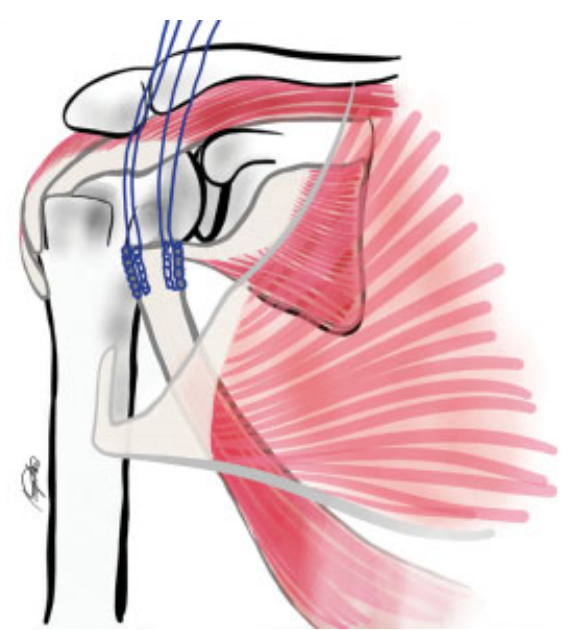

Fig. 3 Desenho do enxerto do GD com passagem de duas linhas de sutura do tipo Krakow com fio Ethibond número 5.

menor, e, em alguns casos, na porção anterior do tubérculo maior (-Figura 4). Mais alguns pontos transósseos são dados, fixando o tendão do grande dorsal à cabeça umeral se necessário, para que haja uma coaptação adequada do enxerto em sua maior superfície no leito ósseo. O coto do subescapular é reparado na borda medial ou sob o tendão do grande dorsal transferido, numa tentativa de melhorar a

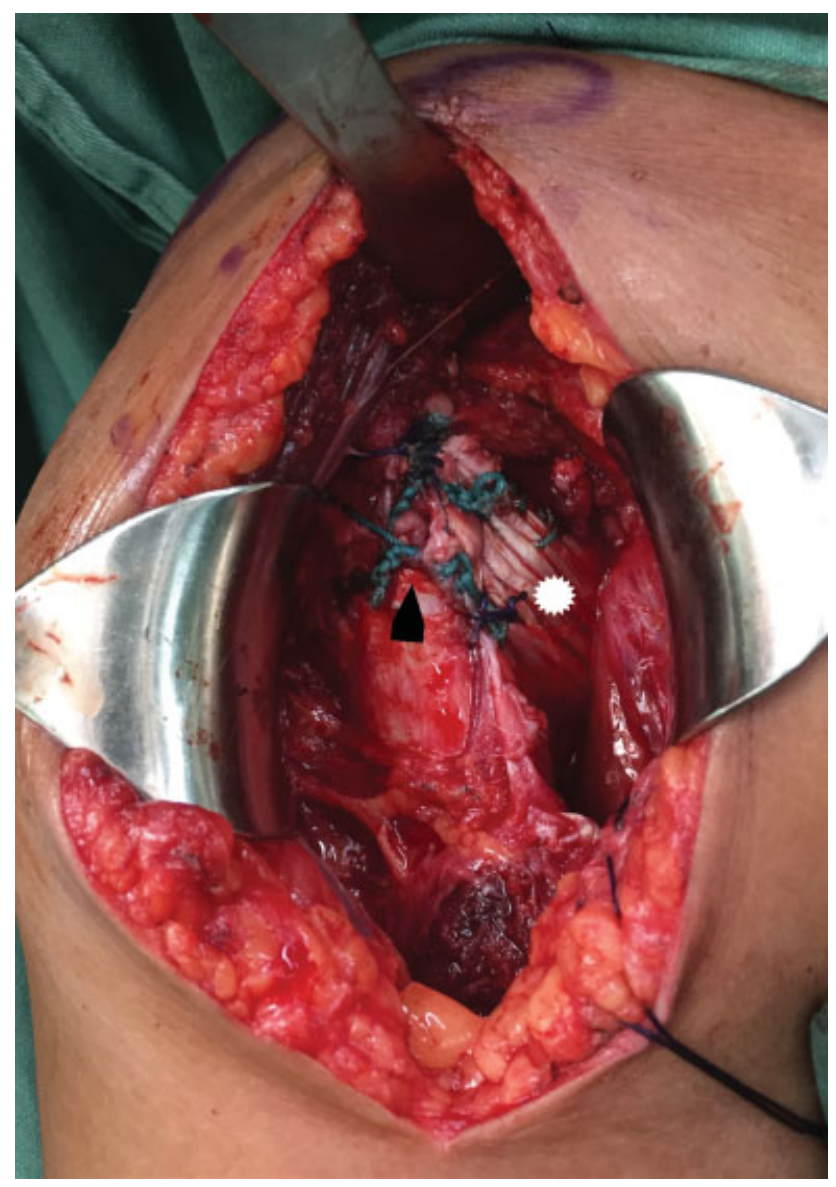

Fig. 4 Foto transoperatória da inserção do enxerto (GD) (asterisco branco) no tubérculo menor do úmero (seta preta). 




Fig. 5 Desenho do esquema final de sutura do enxerto inserido no tubérculo menor do úmero, reparo de lesão anterior do supraespinhal, reparo do coto do subescapular no GD, e sutura da porção tenotomizada do peitoral maior.

tensão da parede anterior do manguito. Caso haja possibilidade, é realizado o reparo de outras lesões de manguito (supraespinhal e infraespinhal) pela mesma via, também com pontos transósseos (-Figura 5). Por fim, é feita a sutura da parte proximal do peitoral maior tenotomizado (-Vídeo 1). Após o fechamento por planos e o curativo, o paciente é imobilizado com uma tipoia com coxim abdutor desenvolvida pelo Grupo de Cirurgia do Ombro para a manutenção do membro em posição neutra funcional. Um vídeo demonstrativo da técnica está no link a seguir (-Vídeo 1).

\section{Vídeo 1}

Técnica de transferência do GD em caso de lesão anterossuperior do manguito rotador. Lesão irreparável do subescapular e lesão reparável do supraespinhal.

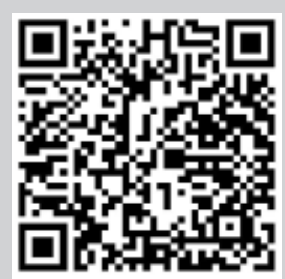
Vídeo em cores. Conteúdo online incluindo sequências de vídeo disponíveis em: https://www.thieme-connect. com/products/ejournals/html/10.1055/s-00411726060.

O manejo pós-operatório é feito com manutenção da tipoia por quatro a seis semanas. Durante esse período, o paciente é orientado a realizar a flexo-extensão do cotovelo com o braço ao lado do corpo já no primeiro dia de pósoperatório. Após a retirada da tipoia, orienta-se a realização de movimentos passivos, e o paciente evolui para mobilidade ativa. Exercícios de reforço muscular são recomendados apenas após a $12^{\circ}$ semana de pós-operatório.

\section{Considerações Finais}

A transferência do grande dorsal em lesões anterossuperiores irreparáveis do manguito rotador (-Vídeo 1) é uma técnica nova que demonstrou ser viável, de baixo custo e sem complicações pós-operatórias em curto prazo. Além disso, pacientes tratados por essa técnica relataram alta taxa de satisfação, e também foi possível utilizá-la em pacientes que apresentavam lesões posterossuperiores associadas. Estudos futuros são necessários para a avaliação da eficácia e da reprodutibilidade da técnica quando comparada a outros métodos de tratamento, assim como seus resultados clínicos em longo prazo.

\section{Suporte Financeiro}

Não houve suporte financeiro de fontes públicas, comerciais, ou sem fins lucrativos.

\section{Conflito de Interesses}

Os autores declaram não haver conflito de interesses.

\section{Agradecimentos}

Ao dr. Augusto Medaglia, pela elaboração e desenho das ilustrações referentes à técnica cirúrgica, e ao dr. Rodrigo Py Gonçalves Barreto, pela colaboração no processo de revisão e formatação do manuscrito.

\section{Referências}

1 Resch H, Povacz P, Ritter E, Matschi W. Transfer of the pectoralis major muscle for the treatment of irreparable rupture of the subscapularis tendon. J Bone Joint Surg Am 2000;82(03): 372-382

2 Omid R, Lee B. Tendon transfers for irreparable rotator cuff tears.J Am Acad Orthop Surg 2013;21(08):492-501

3 Kany J, Guinand R, Croutzet P, Valenti P, Werthel JD, Grimberg J. Arthroscopic-assisted latissimus dorsi transfer for subscapularis deficiency. Eur J Orthop Surg Traumatol 2016;26(03): 329-334

4 Greenspoon JA, Petri M, Warth RJ, Millett PJ. Massive rotator cuff tears: pathomechanics, current treatment options, and clinical outcomes. J Shoulder Elbow Surg 2015;24(09): 1493-1505

5 Konrad GG, Sudkamp NP, Kreuz PC, Jolly JT, McMahon PJ, Debski RE. Pectoralis major tendon transfers above or underneath the conjoint tendon in subscapularis-deficient shoulders. An in vitro biomechanical analysis. J Bone Joint Surg Am 2007;89(11): 2477-2484

6 Moroder P, Schulz E, Mitterer M, Plachel F, Resch H, Lederer S. Long-Term Outcome After Pectoralis Major Transfer for Irreparable Anterosuperior Rotator Cuff Tears. J Bone Joint Surg Am 2017; 99(03):239-245

7 Elhassan B, Ozbaydar M, Massimini D, Diller D, Higgins L, Warner JJ. Transfer of pectoralis major for the treatment of irreparable tears of subscapularis: does it work? J Bone Joint Surg Br 2008;90 (08):1059-1065

8 Elhassan B, Christensen TJ, Wagner ER. Feasibility of latissimus and teres major transfer to reconstruct irreparable subscapularis tendon tear: an anatomic study. J Shoulder Elbow Surg 2014;23 (04):492-499 